\title{
A Report on influences from superfluid turbulence in Der Rufer for percussion quartet
}

\section{Un rapport sur les influences de la turbulence superfluide dans Der Rufer pour quatuor de percussions}

\author{
Michael Edward Edgerton ${ }^{1}$ \\ ${ }^{1}$ Malmö Academy of Music, Lund University, Sweden
}

ABSTRACT. This report will discuss the influence of research into superfluid turbulence on my composition Der Rufer for percussion quartet. Written for Professor Olaf Tzschoppe, director of the Bremen Percussion Quartet, this work is the first result of a planned long-term collaboration with J.G. Weisend II, a Deputy Head of Accelerator Projects at the European Spallation Source (E.S.S.).

KEYWORDS. European Spallation Source (E.S.S.), Superfluid turbulence, Music composition, Art-science collaboration, percussion instruments.

\section{Introduction}

Der Rufer, for percussion quartet was written for and dedicated to Olaf Tzschoppe and the Bremer Schlagzeugensemble and is the first result of a planned, long-term collaboration between Lund University/Malmö Academy of Music and the European Spallation Source (E.S.S.). The European Spallation Source (ESS) is a multi-disciplinary research facility based on the world's most powerful neutron source. Their goal is to enable scientific breakthroughs in research related to materials, energy, health and the environment, and addressing some of the most important societal challenges of our time. In the first step of this cooperation, I collaborated with J.G. Weisend II, a Deputy Head of Accelerator Projects at E.S.S. looking at the issues surrounding Superfluid Turbulence.

The title, Der Rufer refers to a sculpture in Bremen by Gerhard Marcks that refers to a Greek Herald from Homer's Iliad. On the base of the sculpture of written:

Der Rufer von Gerhard Marcks (1889 - 1981)

Der Rufer ist der Figur des Stentor nachempfunden, der mit großherziger und ehernen Stimme so laut rief wie fünfzig Männer. (Homer, Ilias, 730 v. Chr.)

Der Rufer is modeled on the figure of the stentor (Herald, bard, or crier) who shouted as loudly as fifty men in a generous and brazen voice. (Homer, Iliad, 730 BC)

Die drei Meter hohe Bronzeskulptur wurde 1967 von Gerhard Marcks im Auftrag von Radio Bremen geschaffen. Am 25 November 2007 wurde der Rufer vor dem Neubau von Radio Bremen an der Weser aufgestellt.

The three-meter-high bronze sculpture was created in 1967 by Gerhard Marcks on behalf of Radio Bremen. On November 25, 2007, Der Rufer was placed in front of the new Radio Bremen building on the Weser river.

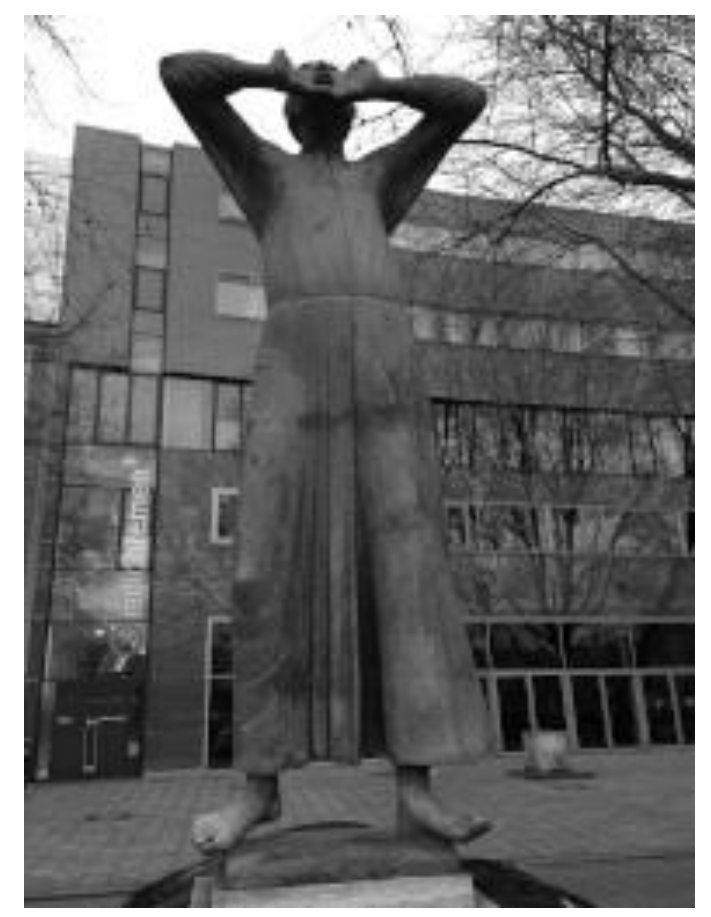


In this report I will present an overview of different aspects of superfluid turbulence that influenced the writing of Der Rufer. Then, I will discuss how those ideas were loosely modelled in this composition. For some time, I have been interested in the motions of turbulence in fluids and was intrigued to enter into discussions about perhaps one of the greatest discoveries in physics in the first half of the $20^{\text {th }}$ century, that of superfluidity ${ }^{1}$. Superfluid occurs when liquid helium is cooled to within 2.172 degrees of absolute zero (absolute zero is about $-273^{\circ} \mathrm{C}$ ). In this ultra-cool region, helium can flow without viscosity or friction, but seldom without turbulence. As I learned through discussions and readings of the literature, superfluidity refers to the flow of superfluid liquid helium $\left(\mathrm{H}_{\mathrm{II}}\right)$ through a tube or pipe that has no friction, no viscosity. Let's compare for a second that of normal helium fluid flow that has friction near the walls of a tube or pipe and thus viscosity. An important property for many mechanical systems, including musical instruments, viscosity refers to the measure of the resistance of some element to deformation, or how thick or sticky something is. For example, syrup is higher in viscosity than water. This is how most fluids (including airflow) react in the real world.

But, superfluids with zero viscosity are extremely rare. When a fluid is produced with zero viscosity this means the fluid can flow without any loss of kinetic energy. What this means is that if you set down a cup of coffee with the liquid moving about and you come back 10 minutes later, the liquid will have stopped moving, since atoms in the liquid will collide with one another and slow down. But if you did that with superfluid helium and come back 10 years later, the liquid will still be moving.

Superfluids feature other amazing behaviors, like the ability of a fluid to flow up the side of a wall or to fit into and flow through impossibly small, molecular-wide spaces, or to remain steady when a container is spun, or to produce a frictionless fountain that will flow forever ${ }^{2}$. Next I will discuss eight principles involved in ultra-cold physics that influenced the composition of Der Rufer.

\section{Materials}

The first property of superfluid turbulence that influenced the composition of Der Rufer is known as anisotropy.

1. ANISOTROPY, is the property of substances to exhibit variations in physical properties along different molecular axes, or in other words, looks different from different perspectives ${ }^{3}$. For example, the viewpoint along the tube (A) is denser than the viewpoint from the side of the tube (B).
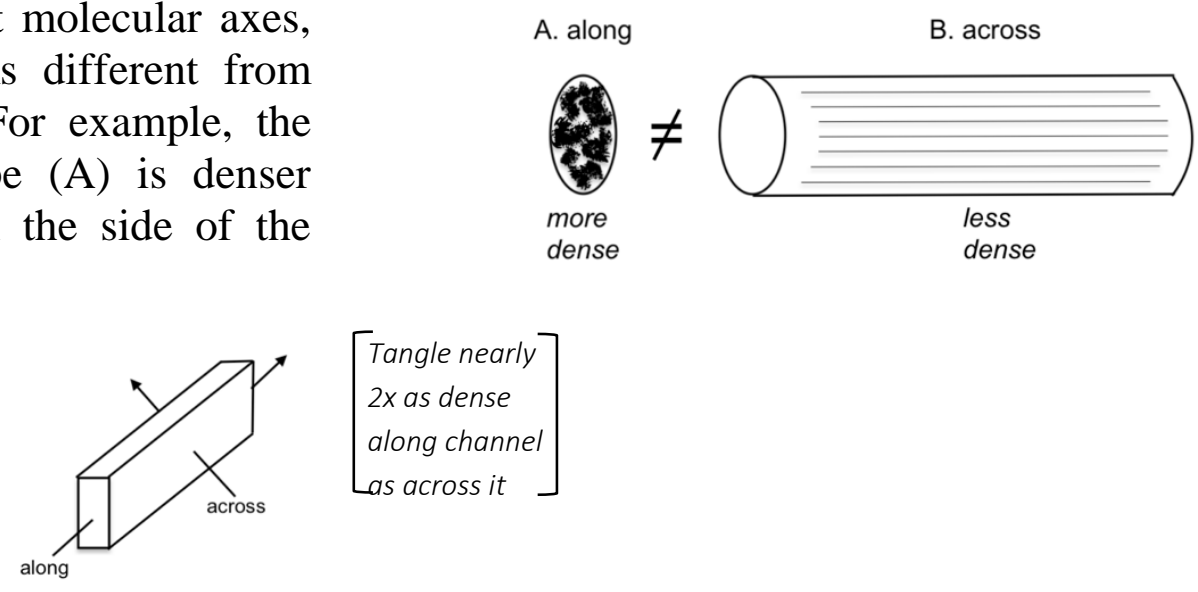

The second property of superfluid turbulence that influenced the composition of Der Rufer is known as isotropy. 
2. ISOTROPY, is the property of substances to exhibit uniformity in all orientations.

Homogenous, not isotropic

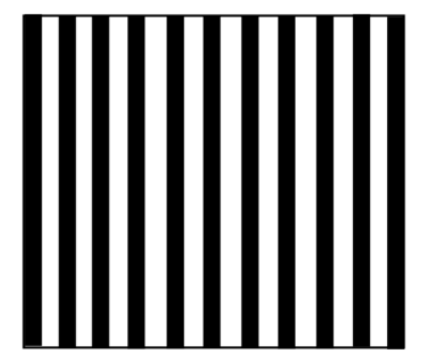

Isotropic, not homogenous

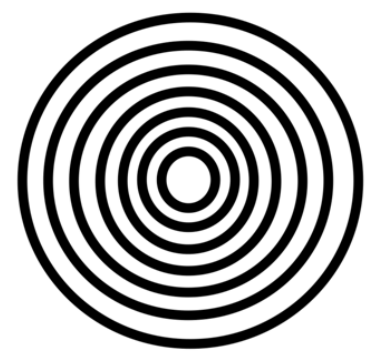

In nature, all flows are anisotropic. Nevertheless, much research has shown that small scale turbulence is modelled successfully using isotropic properties. The reason is that nonlinear fluid flow tends to resemble isotropy when cascading at smaller scales. However, with superfluid turbulence we move into conditions that promote quasi two-dimensional flow that will be discussed further with the property known as second sound ${ }^{4}$.

The third property of superfluid turbulence that influenced my work in Der Rufer were vortices.

3. VORTEX, is the property of fluids to form a spiral. In fluid, a vortex revolves around an axis line, which may be straight or curved. Vortices may be seen in a cup of coffee, in the draining of a bathtub, in a tornado or in the turbulent wake following an airplane. Vortices can move, stretch, twist and interact in complicated ways and are important in turbulence. The velocity within a vortex is greatest next to its core and decreases with distance from its center ${ }^{5}$.

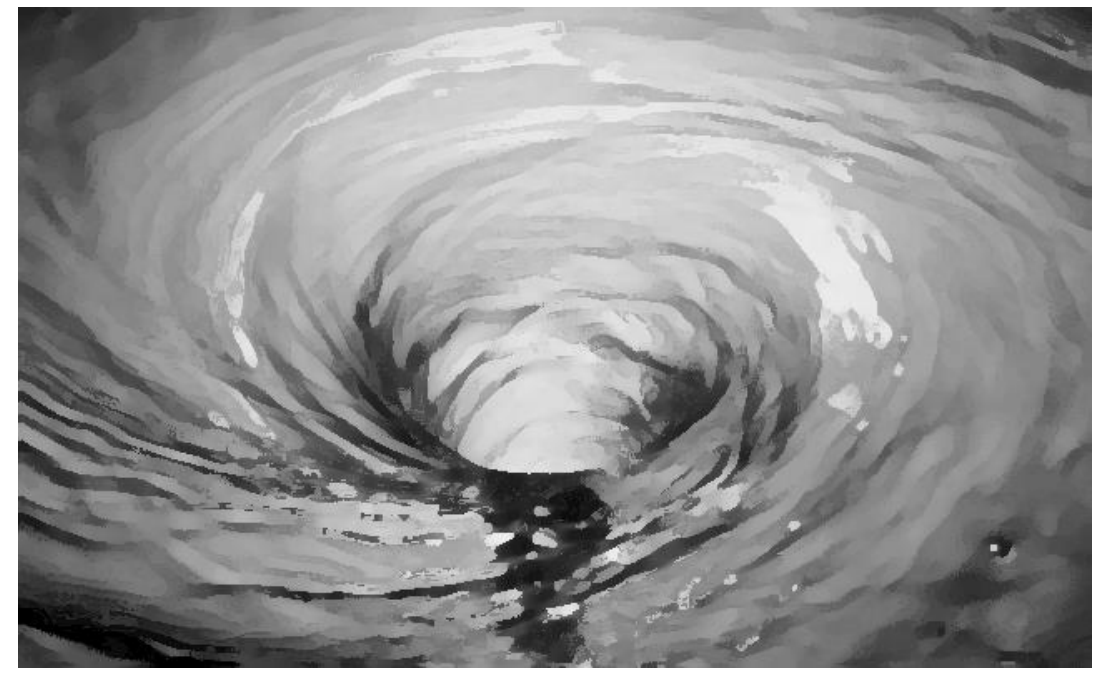

However, in ultracold superfluids, atoms can be controlled very precisely and thus bring to life complicated mathematical equations in order to test whether they work in the real world. Since the 1990s physicists have studied many models in ultracold experiments, pushing the boundaries in every direction imaginable. This includes work with vortices, which turn out to be ideal building blocks of dynamical superfluid flows which are helping to understand the larger questions of quantum turbulence ${ }^{6}$, such as damping and dissipation ${ }^{7}$.

The fourth property of superfluid turbulence that influenced my work in Der Rufer is known as vortices, bending and twisting.

4. VORTICES, Bend and Twist. In the 1950s, Feynman proposed that superfluids rotate in more complicated ways than normal fluid. When both fluids are generated in a bucket, interactions between normal fluid and superfluid appear when the counterflowing velocity is high. The resultant 
quantized vortices interfere with the counterflowing fluid, which when displaced can bend and twist cores as normal and superfluids come within a critical bandwidth of any vortex ${ }^{3}$.

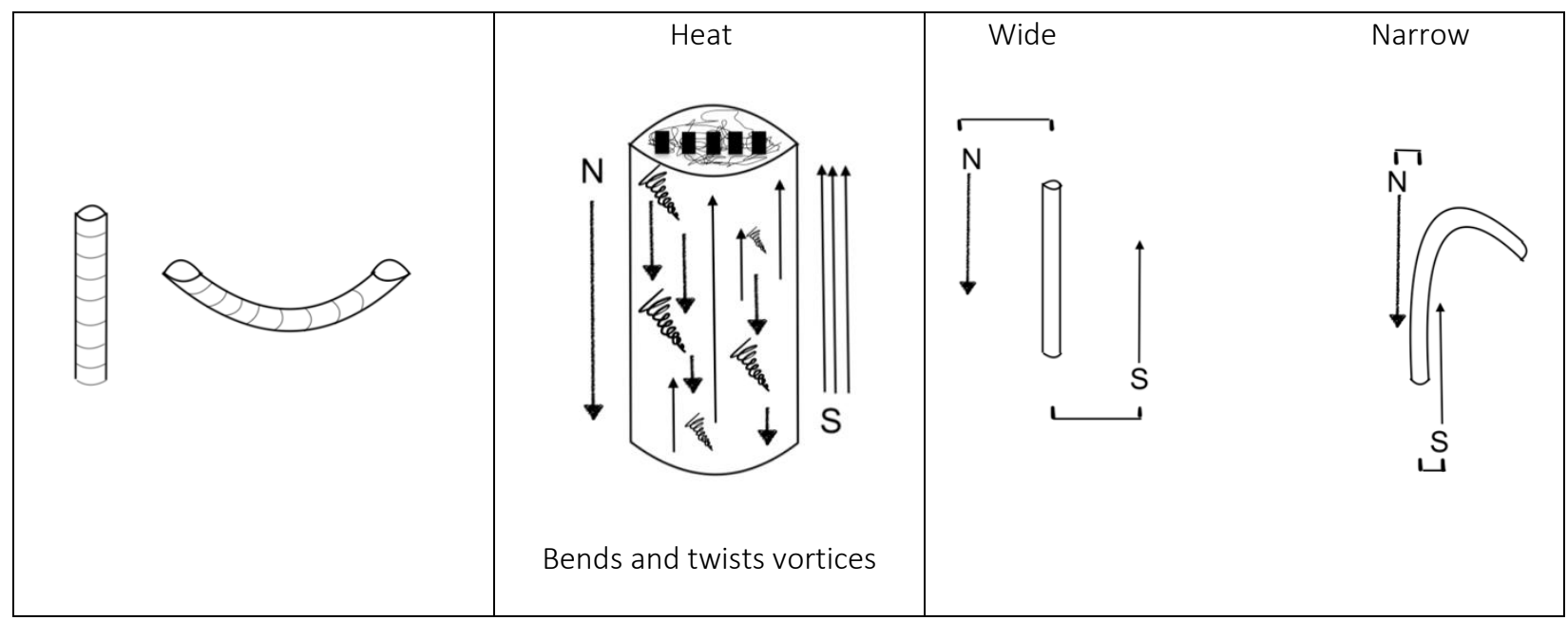

The fifth property of superfluid turbulence that influenced my work in Der Rufer is known as quantum leap.

5. BIFURCATION (quantum leap). In quantum mechanics, an atom must occupy only one energy level. As the atom moves about, it can only acquire energy in set amounts. In order to move to a higher energy state, only a relatively high energy disturbance may drive the atom into a higher state. Generally, this can happen when energy from light or electricity acts on particles ${ }^{8}$.

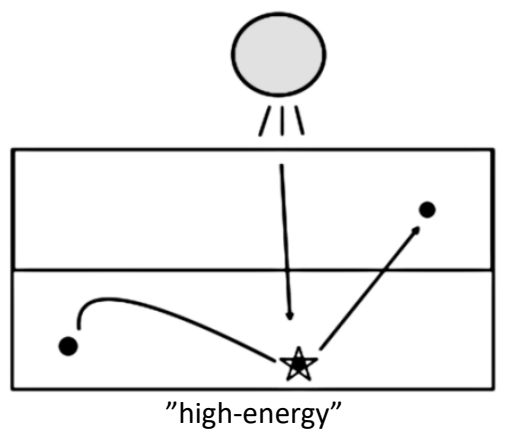

The sixth property of superfluid turbulence that influenced my work in Der Rufer is known as mutual friction.

6. MUTUAL FRICTION, occurs when normal fluid and superfluid are coupled, where velocity and temperature are higher ${ }^{9}$. As friction expands beyond normal fluid to affect superfluid flow, the superfluid begins to extract energy from normal fluid and grow in amplitude ${ }^{10}$. Ordinarily, normal fluid and superfluid flow in opposite directions where normal fluid has some friction, while superfluid has no friction, and normal fluid \& superfluid flow past each other freely. However, during mutual friction we find coupling between normal fluid and superfluid in the center of a channel, so that they drag each other around. At some point, the coupled vortices are pushed to the boundary, where increased friction at the walls create a turbulent layer - almost a random tangle ${ }^{10}$.
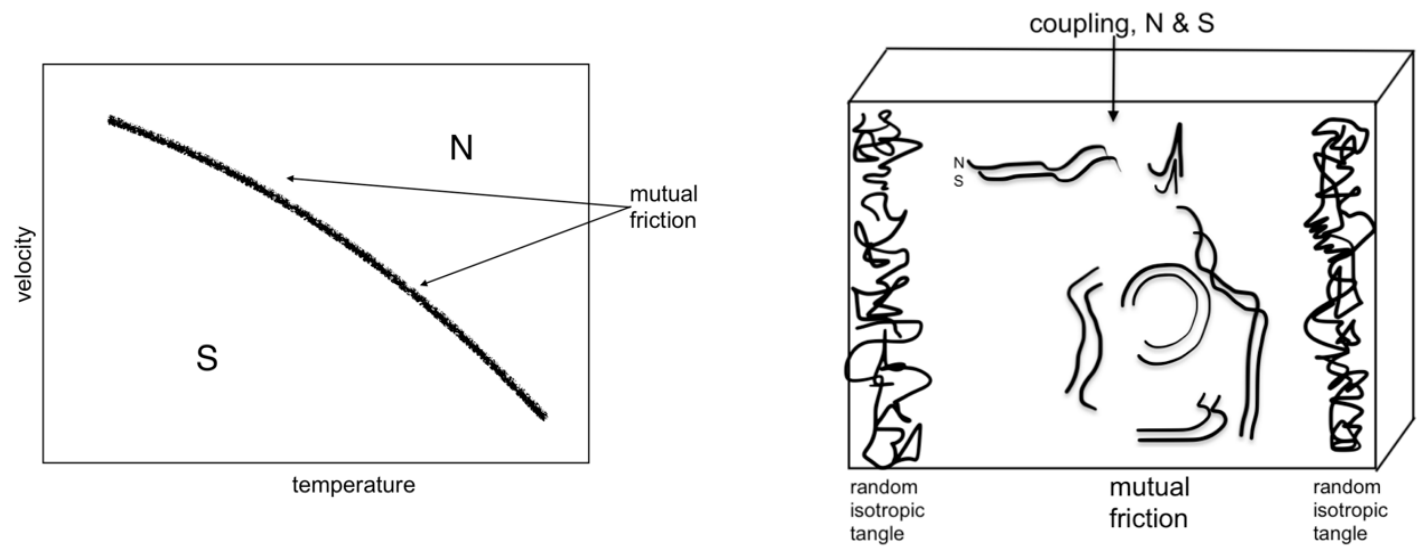
The seventh property of superfluid turbulence that influenced my work in Der Rufer is known as turbulence past an obstacle.

7. TURBULENCE, Past an Obstacle.

In normal fluid, turbulence often occurs past an obstacle, like large boulders in a rapids, in the manner of eddies, whirlpools and all manner of vortices. Turbulence decays downstream of the obstacle ${ }^{3}$

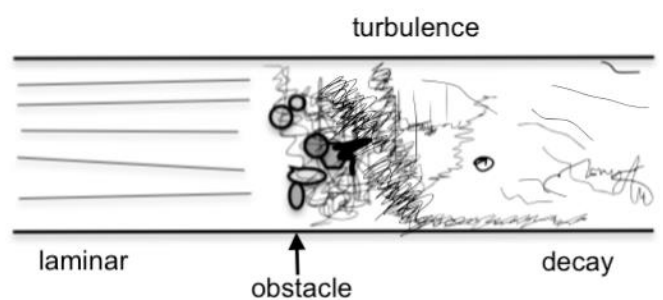

The eighth property of superfluid turbulence that influenced my work in Der Rufer is known as second sound.

8. SECOND SOUND, is a quantum mechanical phenomenon which propagates through superfluid helium as a temperature wave rather than as a dispersal of energy. It is known as second sound because the wave motion of heat is similar to the wave motion of sound, which consist of fluctuations in the density or pressure of molecules in air. Similarly, second sound consists of fluctuations in the density of particle-like thermal excitations (rotons and phonons) in superfluid.

Another important property of second sound is that superfluid helium consists of two fluid elements that can flow independently of one another. Normal fluid and supersound flow in opposite directions and thus "flow

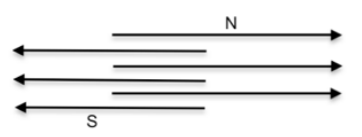
through each other'.

At low temperatures, viscosity disappears in superfluid, contrary to normal fluid where viscosity increases as temperature decreases. Some of the interesting effects from superfluid helium include a fountain that may flow forever due to thermomechanical properties of superfluid with no viscosity; that superfluid helium may climb up the sides of a bucket, and; that in a two-fluid experiment, that normal helium can be trapped by a plate with micro-holes while when cooled to a superfluid, helium can pass through the micro-holes.
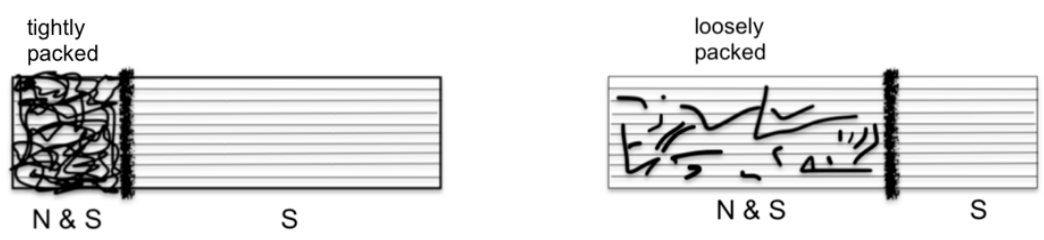

Even though the regions of high and low density of normal fluid and superfluid change over time, the overall density remains the same ${ }^{11,12,13,14}$.
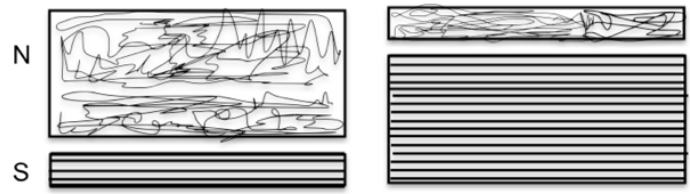

\section{Interpretation}

In this second half of the paper, I will discuss how the different concepts from the study of superfluid influenced the process or materiality of the music in Der Rufer.

1. ANISOTROPY. In the material influenced by anisotropy, I focused on two ideas. The first was that a property will look different from a different perspective, while the second idea was to develop textures that were heterophonic.

$1^{\text {st }}$ appearance. A single rhythmic series served as the object viewed from different perspectives. The idea was that this entity was viewed from three different positions. These differing points of view exhibited: 
A. A contradiction; in which active, expressive gestures played over large physical distances on marimba and vibraphone, are muted in order to lessen the sense of pitch almost completely. The instruments decouple slightly from the monophonic rhythmic series, mirroring entropic processes, which at the end of this first section appears in retrograde.

B. A less dense materiality is seen when the guiro (a musical instrument of the idiophone family, common in Cuba and Puerto Rico, consisting of a scraper with holes through which the thumb and middle finger are passed to hold it), plucked with a plastic card, is paired with a damped ratchet (a percussion instrument with a gearwheel and stiff board mounted on a handle, which rotates freely). This material produces a sequence of soft, staccato and unpitched percussive sounds.

C. La bourgeoisie - a middle of the road gesture, musically akin to a social class oriented to economic materialism and hedonism, here due to the malletwork on two damped and unpitched metal bars and woodblocks. The coupling with the monophonic rhythmic line is nearly $100 \%$, as is also played out in capitalist rhetoric. I

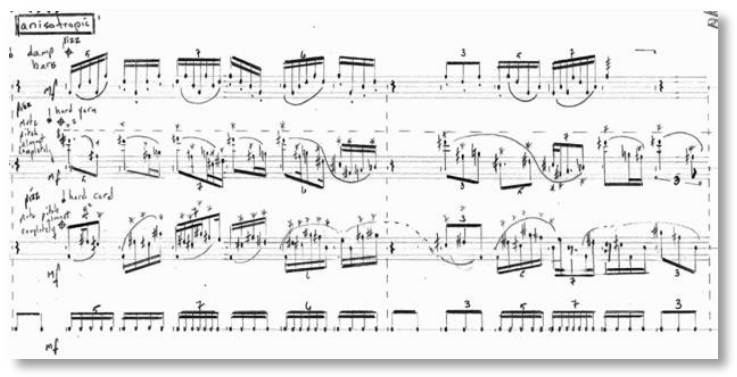
propose that these elements may be viewed from different perspectives due to their muted and/or compressed characters in this drama.

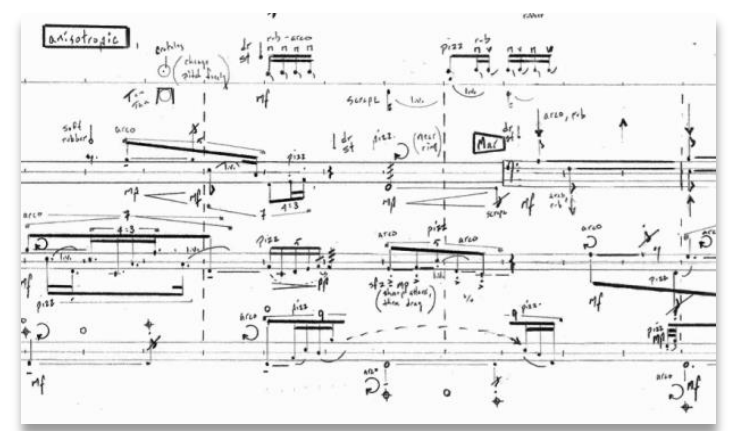

$2^{\text {nd }}$ appearance. The second anisotropic section is in two parts. The first part is heterophonic, presenting a fourth perspective on the single rhythmic series, which here is viewed directly adjacent to the core rhythmic series, after its demolition. The result is a complicated tangle of events, including unusual sustaining procedures, such as producing an arco vibraphone tone by rubbing a drumstick longitudinally along the bar length, with moderately high pressure (arco usually refers to when bowed string instruments sustain a tone through continuous up and down bow strokes on a string). The second part is essentially a return to the expression of the $1^{\text {st }}$ appearance of anisotropy, with the addition of struck and lightly damped crotales.

2. ISOTROPY. In the material influenced by isotropy I focused on two ideas. The first was that a property will look uniform in all orientations, while the second was that the material would assume a homophonic texture.

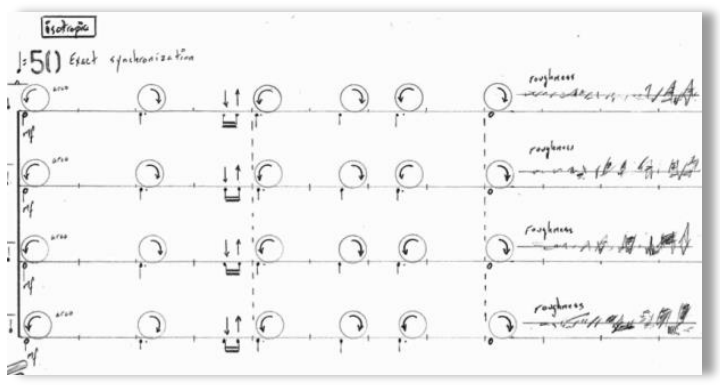

Isotropy is loosely modelled in a single section of homophonic material. In this section, all performers are asked to perform "true" sustained tones by rubbing a handheld cymbal with drumstick (arco-rub). Referencing orchestral string performance practice, the percussionists are asked to exactly synchronize arco-rub similar to synchronized bowing by orchestral string sections, changing up- or down-bow simultaneously. As well, the players are asked to produce timbral changes on the cymbals that include playing pure tones, increase/decrease of roughness, vary pressure and scrape, changing direction of rub, etc. 
3. VORTEX. In the sections influenced by vortices I focused on the single idea of the revolving cone-like phenomena seen in whirlpools and tornados, etc. Written for vibraphone (with supplemental metal bars) and marimba (with supplemental wood sticks), these materials based on vortices reveal the most complicated pitch and non-determinative rhythmic structures in the entire piece.

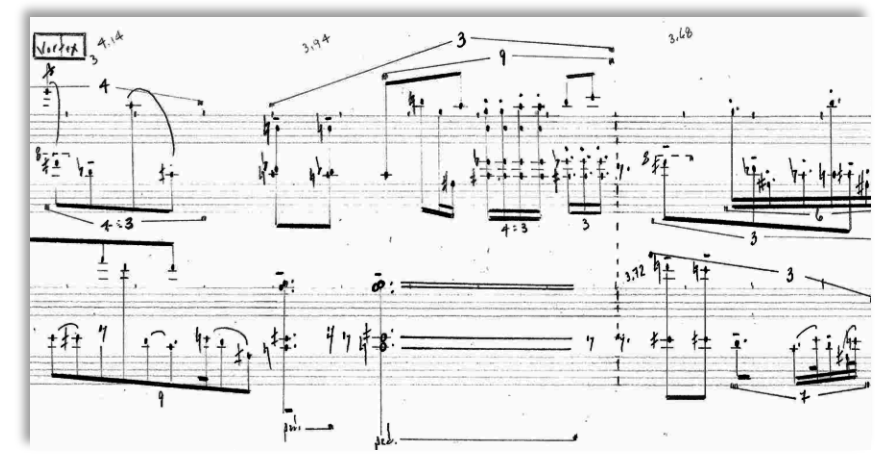

A table of integers was developed to generate nondeterminative rhythms. These numbers were derived from measures of the physical distance between nodes on the boundary of the largest vortex I modelled. The measurements were taken at a descending series of nodes, for example the distance between point $\mathrm{A}$ and point $\mathrm{B}$ equaled $14.5 \mathrm{~cm}$; while the distance between point $\mathrm{B}$ and point $\mathrm{C}$ equaled $13.8 \mathrm{~cm}$, etc. In total there were 17 points which produced 16 measurements. In order to build in variation and develop scaling that would allow for intuitive control, I transposed this initial integer series downward, so that

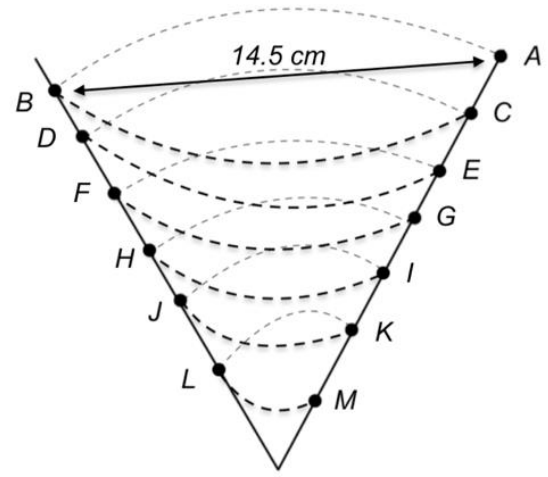
there were 21 integer series in total.

\section{$\begin{array}{llllllllllllllllllll}1.1 & 1.3 & 1.5 & 1.7 & 1.9 & 2.1 & 2.3 & 2.5 & 2.7 & 2.9 & 3.1 & 3.3 & 3.5 & 3.7 & 3.9 & 4.1 & 4.3 & 4.5 & 4.7 & 4.9\end{array}$}

\begin{tabular}{|c|c|c|c|c|c|c|c|c|c|c|c|c|c|c|c|c|c|c|c|c|}
\hline 14.5 & 13.18 & 11.15 & 9.66 & 8.5 & 7.63 & 6.9 & 6.3 & 5.8 & 5.37 & 5 & 4.67 & 4.39 & 4.14 & 3.91 & 3.7 & 3.5 & 3.37 & 3.22 & 3.08 & 2.95 \\
\hline 13.8 & 12.54 & 10.61 & 9.2 & 8.11 & 7.26 & 6.57 & 6 & 5.52 & 5.11 & 4.75 & 4.45 & 4.18 & 3.94 & 3.72 & 3.53 & 3.36 & 3.2 & 3.06 & 2.93 & 2.81 \\
\hline 12.9 & 11.72 & 9.92 & 8.6 & 7.58 & 6.78 & 6.14 & 5.6 & 5.16 & 4.77 & 4.44 & 4.16 & 3.9 & 3.68 & 3.48 & 3.3 & 3.14 & 3 & 2.86 & 2.74 & 2.63 \\
\hline 11.85 & 10.77 & 9.11 & 7.9 & 6.97 & 6.23 & 5.64 & 5.15 & 4.74 & 4.38 & 4.08 & 3.82 & 3.59 & 3.38 & 3.2 & 3.03 & 2.89 & 2.75 & 2.63 & 2.52 & 2.41 \\
\hline 10.7 & 9.72 & 8.23 & 7.13 & 6.29 & 5.63 & 5.09 & 4.65 & 4.28 & 3.96 & 3.68 & 3.45 & 3.24 & 3.05 & 2.89 & 2.74 & 2.6 & 2.48 & 2.37 & 2.27 & 2.18 \\
\hline 9.52 & 8.65 & 7.32 & 6.34 & 5.6 & 5.01 & 4.53 & 4.13 & 3.8 & 3.52 & 3.28 & 3.07 & 2.88 & 2.72 & 2.57 & 2.44 & 2.32 & 2.21 & 2.11 & 2.02 & 1.94 \\
\hline 8.35 & 7.59 & 6.42 & 5.56 & 4.91 & 4.39 & 3.97 & 3.63 & 3.34 & 3.09 & 2.87 & 2.69 & 2.53 & 2.38 & 2.25 & 2.14 & 2.03 & 1.94 & 1.85 & 1.77 & 1.7 \\
\hline 7.23 & 6.57 & 5.56 & 4.82 & 4.25 & 3.8 & 3.44 & 3.14 & 2.89 & 2.67 & 2.49 & 2.33 & 2.19 & 2.06 & 1.95 & 1.85 & 1.76 & 1.68 & 1.6 & 1.53 & 1.47 \\
\hline 6.18 & 5.61 & 4.75 & 4.12 & 3.63 & 3.25 & 2.94 & 2.68 & 2.47 & 2.28 & 2.13 & 1.99 & 1.87 & 1.76 & 1.67 & 1.58 & 1.5 & 1.43 & 1.37 & 1.31 & 1.26 \\
\hline 5.23 & 4.75 & 4.02 & 3.48 & 3.07 & 2.75 & 2.49 & 2.27 & 2.09 & 1.93 & 1.8 & 1.68 & 1.58 & 1.49 & 1.41 & 1.34 & 1.27 & 1.21 & 1.16 & 1.11 & 1.06 \\
\hline 4.38 & 3.98 & 3.36 & 2.92 & 2.57 & 2.3 & 2.08 & 1.9 & 1.75 & 1.62 & 1.51 & 1.41 & 1.32 & 1.25 & 1.18 & 1.12 & 1.06 & 1.01 & 0.97 & 0.93 & 0.8 \\
\hline 3.63 & 3.3 & 2.79 & 2.42 & 2.13 & 1.91 & 1.72 & 1.57 & 1.45 & 1.34 & 1.25 & 1.17 & 1.1 & 1.03 & 0.98 & 0.93 & 0.88 & 0.84 & 0.80 & 0.77 & 0.74 \\
\hline 2.99 & 2.71 & 2.3 & 1.99 & 1.75 & 1.57 & 1.42 & 1.3 & 1.19 & 1.1 & 1.03 & 0.96 & 0.9 & 0.85 & 0.8 & 0.76 & 0.72 & 0.69 & 0.66 & 0.63 & 0.61 \\
\hline 2.44 & 2.21 & 1.87 & 1.62 & 1.43 & 1.28 & 1.16 & 1.06 & 0.97 & 0.9 & 0.84 & 0.78 & 0.73 & 0.69 & 0.65 & 0.62 & 0.59 & 0.56 & 0.54 & 0.51 & 0.49 \\
\hline 1.98 & 1.8 & 1.52 & 1.32 & 1.16 & 1.04 & 0.94 & 0.86 & 0.79 & 0.73 & 0.68 & 0.63 & 0.6 & 0.56 & 0.53 & 0.5 & 0.48 & 0.46 & 0.44 & 0.42 & 0.4 \\
\hline 1.6 & 1.45 & 1.23 & 1.06 & 0.94 & 0.84 & 0.76 & 0.69 & 0.64 & 0.59 & 0.55 & 0.51 & 0.48 & 0.45 & 0.43 & 0.41 & 0.39 & 0.37 & 0.35 & 0.34 & 0.3 \\
\hline
\end{tabular}


Five three-dimensional vortices were modelled and then mapped upon a twodimensional space, where the relative height of each point was translated into pitch. However, for time and rhythm, I did not develop a new matrix for each vortex, as it was important to build in as much redundancy as possible, while still retaining a certain level of real-world complexity.

How did I translate numbers into rhythm? Below I show three examples of this process. Important for me was the build into the process were procedures that allowed for intuition and interpretation. If we look at the table above, at the series beginning with 4.14 and descending to 0.45 .

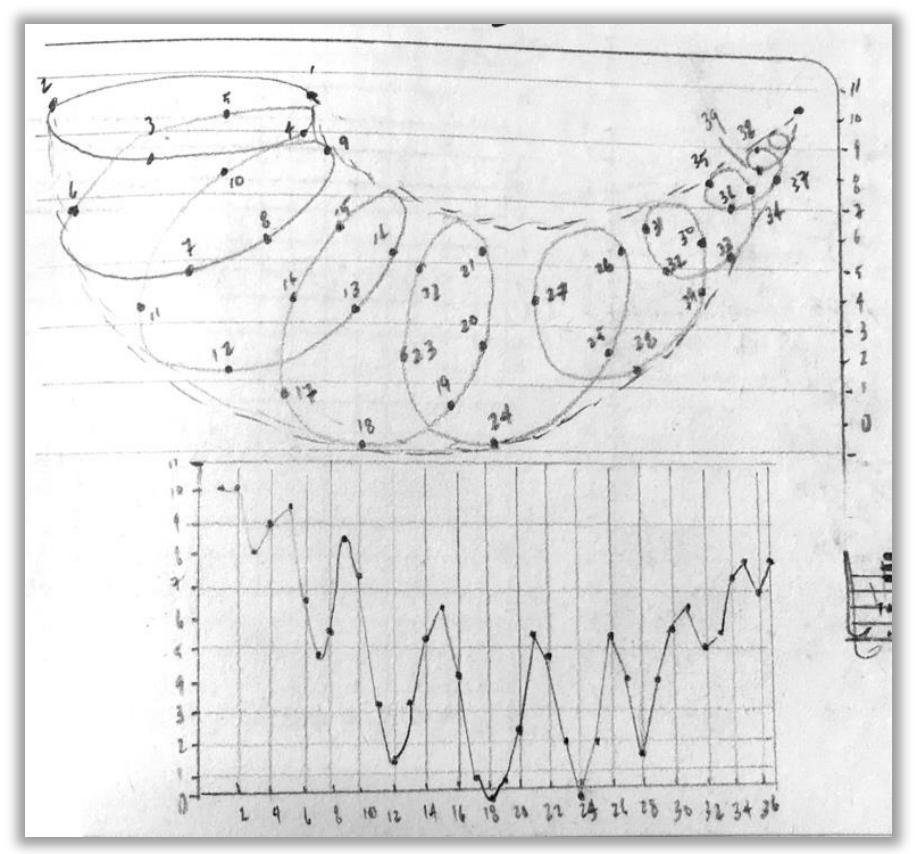

The process involved choosing each box in a descending order, so for instance, beginning at 4.14 then moving to 3.94 , then to 3.68 , etc. Then in order to interpret each number sequence, I followed the general process:

How did integers become rhythms?

I. Generally, the first integer defined the division/subdivision or iteration of the allotted timespan

II. the second integer defines one of the pre-final sub-units

III. the last integer defines the closing of the gesture

Letter A presents an interpretation of 3.94.

The first number (3) identified a division or iteration of the time span allotted to the figure. For example, look at letter A, here the numbers used were 3.94. As we can see the overall length of the timespan is divided by a triplet. Next, the second number (9) is asked to control one of the pre-final sub-units. So, in example A, we see that the first pulse is a single half-note. This is followed by 9-tet (9:2) spanning the second and third elements of the triplet. Then, the third number (4) defines the closing part of the gesture. In this example, the final number becomes a 4:3 tuplet. In this process, I've embedded the requirement that I must work out of these figures in a very active way in order to require intuition at every step of working out the matrix. This figure would be an example of a determinative rhythm. 
Letter B presents an interpretation of 4.39 that features an extension of steps $1-3$. In this case, the first number (4) identifies the division or iteration of the time span allotted to the figure. Next, the second number (3) controls one of the pre-final sub-units, identified as the triplet ending with a l.v. Then, the third number (9) defines the closing of the gesture. However, this figure breaks the rule by extending beyond the allotted timespan for this gesture and functions as a quasiindependent expression. This figure would be an example of

B.

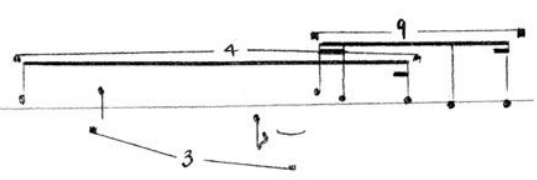
a non-determinative rhythm.

4. VORTICES, Bend and Twist. In the material influenced by vortices, bend and twist, I focused on the idea that normal and superfluid flows can bend and twist vortex cores if/when they come within a critical bandwidth. In Der Rufer, there are three appearances of this influence, which were musically interpreted as unusually sustained legato gestures pairing marimba and vibraphone with voice.

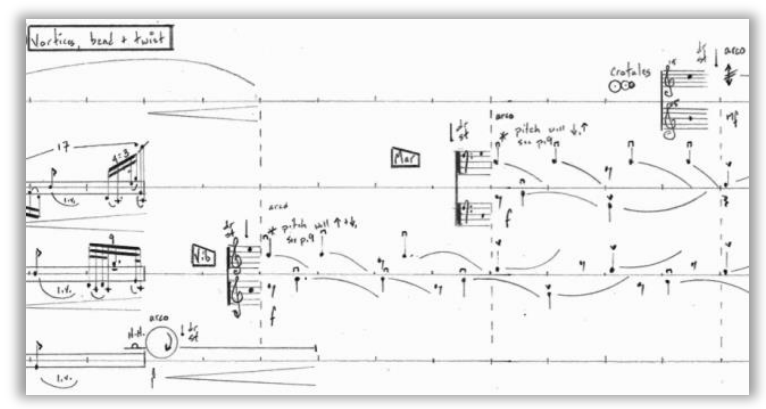

These mallet keyboard instruments are to be performed arco-rub with drumsticks. These actions will produce more or less continuous glissandi that will raise and lower depending on speed, pressure and placement of the stick on the bar. The resultant pitches do not seem to have a simple relationship with the harmonic structure of any selected bar (I'm not aware of any proper acoustical explanation for these continual glissando contours). Accompanying the keyboard instruments, are the crotales (played arco-rub with drum stick), handheld cymbals and Thai gongs (also arco-rub with drum stick).

At times, a voice appears, playing the role of Der Rufer. The voice should be nasal and pressed. In the first appearance of this material, the voice should be in a moderately high tessitura as it mimics the gestures of the vibraphone. In the second appearance of this material, the voice is to mimic the marimba which is played arco-rub with a superball mallet, which will produce pitch contours in a considerably lower tessitura than when vib is rubbed with drumstick. While mimicking the instruments, the voice performer is asked to sing a Greek text (IPA symbols notated) which could be the sort of thing a stentor would intone to his troops on the way to battle. The texts, written by me in Greek, are translated into English below:

"send young man on foot into holy sea, away from war with truth"

"fear not child"

"fear not death"

"work with God"

"write with truth"

In the final section, the voice reappears, again in a higher tessitura, this time accompanying him/herself with a large tom-tom to emphasize and reinforce the strong nature of the message $\mathrm{s} / \mathrm{he}$ is sending. At the end of the piece, all instruments, including the voice become softer. However, the performer is asked not to lose

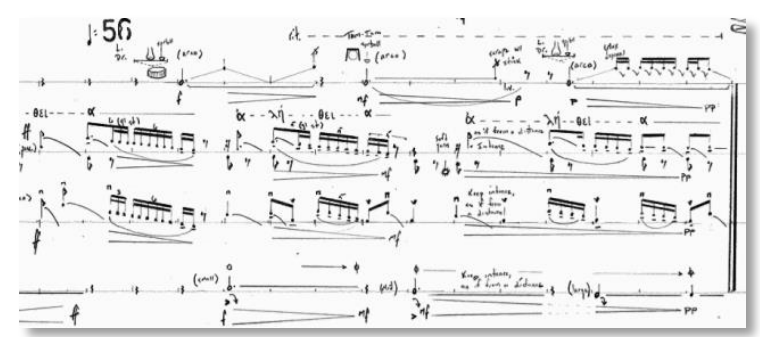

Page | 9 
energy, as the idea here is that the sounds become softer due to distance, as if the soldiers were marching further away in the distance.

5. BIFURCATION (Quantum Leap). In the material influenced by bifurcation (quantum leap), I focused on the idea that in quantum mechanics an atom must occupy only one energy level, and that only high energy disturbances can drive fluid into a higher state.

This material is about energy. All changes of materiality are accompanied by a corresponding change of tempo and dynamic. Since a high energy disturbance is needed to drive the fluid to a higher level, I used transitions between subsections to produce energy changes, for example cresc or decresc with arco-rub on Tibetan cymbal or tam-tam.

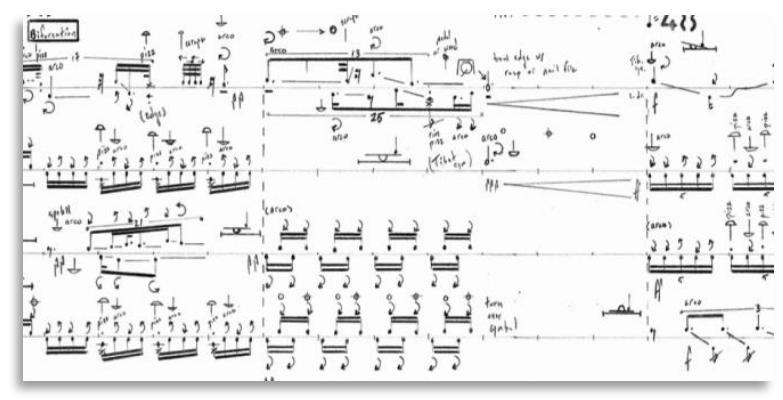

6. MUTUAL FRICTION. In the section influenced by mutual friction, I focused on the idea of attachment between normal fluid and superfluid, so that they drag each other around. In this material, superfluid is represented by arco-rub on handheld cymbals, while normal fluid is represented by rhythmically active pizz and arco gestures on the other instruments. Representing the addition of friction, I've asked the performers to produce timbral changes to the sustained

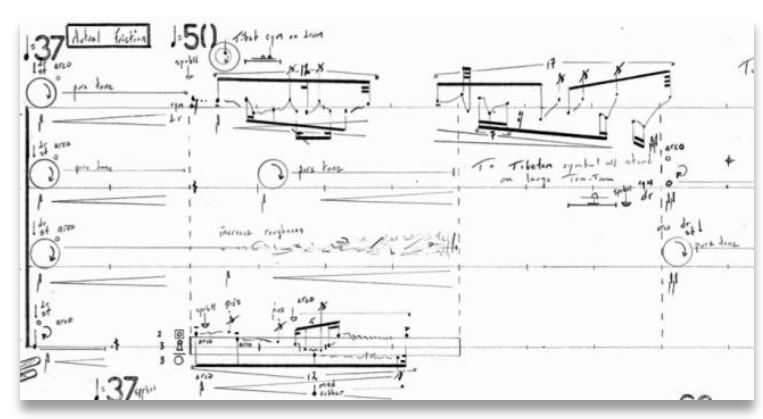
rubbing motions on the handheld cymbals.

7. TURBULENCE, Past an Obstacle. In the sections influenced by turbulence past an obstacle, I focused on the idea that turbulence which occurs downstream of an obstacle progressively decays over time. Beginning with laminar flow produced by arco-rub on handheld cymbals, each section then features the sonic equivalent of an obstacle in a stream in the form of a loud crash. At which point, turbulence occurs in the form of soft rattles, scratches,

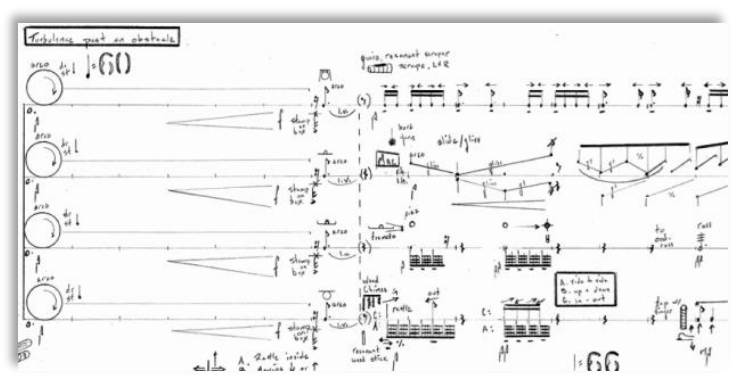
tremolo and all manner of eddies and whirlpools. Adhering to the central idea in these sections, these sounds decay over time.

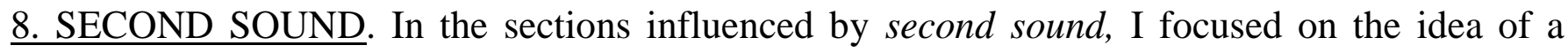
wavelike phenomenon that features alternating regions of densely and loosely packed normal fluid molecules, while the superfluid remains unaffected since $\mathrm{S}$ has no viscosity.

Superfluid is represented by arco-rub on handheld cymbals, while normal fluid is portrayed by pizz and arco production within their corresponding multipercussion setups. The materials were organized around four principles that were mixed and matched including: (1) tightly packed normal fluids, (2) loosely packed normal fluids, (3) normal fluid, featuring high viscosity, (4) superfluid featuring low/no viscosity. A final controlling principle in this gesture is that the overall density must stay constant. Here in example to the right, we see a section in which tightly packed normal fluid (N) in perc 1, 3, 4

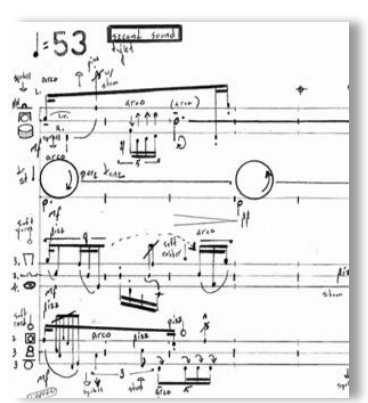
is combined with one part of superfluid $(S)$ in perc 2. After a brief period of fluctuation, this arrangement of $3 \mathrm{~N}$ to $1 \mathrm{~S}$ continues until only $\mathrm{S}$ remains when the normal fluid suddenly disappears, like what happens with ultra-cold fluid as temperatures drop towards absolute zero. 
Following this brief $\mathrm{S}$ material, a loosely packed molecular section appears, which features only normal fluid $(\mathrm{N})$ and no superfluid $(\mathrm{S})$.

In general, the character of the second sound material features heterogeneous textures of rhythmically active (tightly packed) motion versus more static (loosely packed) motion, as well as ; homogeneous textures of normal character versus the superfluid arco-rub character.

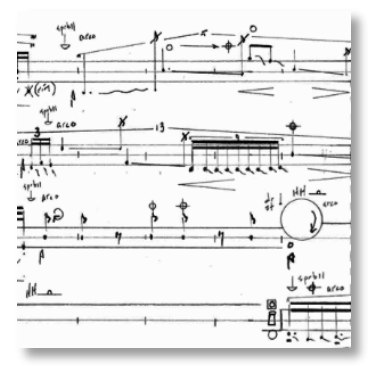

FORM AND STRUCTURE. A note about form and structure. Der Rufer is based on eight interlocking principles from the study of turbulence in normal fluid and superfluid. Each principle serves as the material basis for multiple sections throughout the piece. As these principles are interlocked, so are the sonic properties and their organization. This means that we do not have the perception of eight different styles, nor organizational procedures, etc., but rather a better description would be that the sections organize themselves into overlapping cloud formations, in which, constants are often retained between sections. In these cases, we often have the impression of elision or transition. To be sure there are abrupt shifts that occur in the piece, so the overall effect will be a mixture of evolution with less common bifurcations to distantly related material. This cloud-like function can be visualized in two-dimensions: $1^{\text {st }}$ between chaos and regularity; $2^{\text {nd }}$ between quick and active iterations and slow moving, static elements as is shown below.

As can be seen in the following image, the clouds seem to cluster in three parts. The top cluster features clouds with complex movements trending towards chaos. Anisotropic assumes a lower position due to the overall complexity being reduced as a result of the material existing within a monophonic line and thus less complicated than vortex.

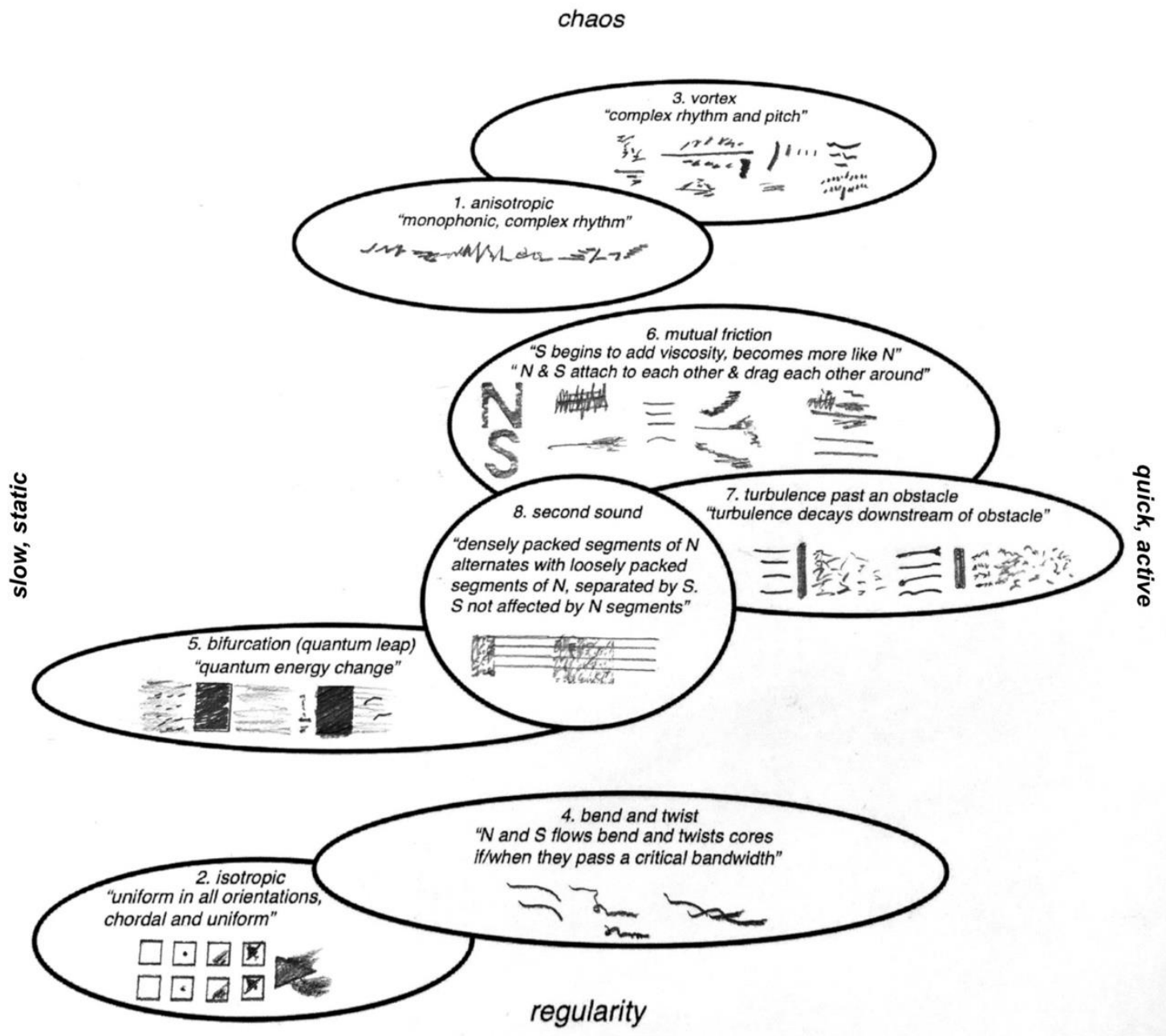


The middle cluster features a mixture of complicated elements combined with more regular materials, due to the exchange of normal fluid with superfluid. Of this cluster the structure of mutual friction would involve the most complex material of this cluster, while the bifurcation of quantum material focuses mostly on energy differences, represented by dynamic/loudness changes.

The lowest cluster brings the listener to a more normal experience in terms of organization and structure. However, during bend and twist, the real-time effect of voice with marimba or vibraphone may, in fact, produce the more unsettling character of nasal voice singing with arco wood or metal than with any of the other sections. The lowest cloud (isotropic) is nearest to absolute regularity, as a kind of chordal production. Here all players play handheld cymbals with drumsticks exactly synchronized in a sustained (arco-rub) manner.

\section{About the author}

I am a composer and currently Professor of Artistic Research in Music at Lund University in Sweden. After completing my Doctorate from the University of Illinois in 1994, I began work as a Postdoctoral Fellow studying vocology \& acoustics through the National Center for Voice and Speech from 1996 to 1999, in order to prepare for writing my book, The $21^{\text {st }}$ Centtury Voice. Creatively, I have long been influenced by interesting thinkers in many fields, including physics. For the collaboration with Dr. Weisend, we shared many long discussions about cryogenics and music which led me to many fascinating articles on superfluid turbulence. Due to Covid restrictions, the premiere has been postponed for a year and is now rescheduled for October 1, 2021 in Germany.

\section{References}

1. R.J. Donnelly. “The Discovery of Superfluidity”. Physics Today $48: 7$ (1995) 30-36.

2. J.R. Minkel. "Strange but True: Superfluid Helium Can Climb Walls". Scientific American 300:2 (2009) N.p. Retrieved 2020-06-17.

3. R.J. Donnelly. “Superfluid Turbulence”. Scientific American 259:5 (1988) 100-109.

4. L. Biferale, D. Khomenko, V. L'vov, A. Pomyalov, I. Procaccia, and G. Sahoo. "Superfluid Helium in ThreeDimensional Counterflow Differs Strongly from Classical Flows: Anisotropy on Small Scales”.

Phys. Rev. Lett. 122:14 (2019) 144501/1-6.

5. Wikipedia contributors. "Vortex." Wikipedia, The Free Encyclopedia. Wikipedia, The Free Encyclopedia, 10 Jun. 2020. Web. 18 Jun. 2020.

6. K. Padavic-Callaghan. “The Coolest Physics You've Ever Heard Of”. Scientific American 322:1 (2020, Jan 20) N.p. Retrieved 2020-06-19.

7. W.F. Vinen, R.J. Donnelly. “Quantum Turbulence”. Physics Today 60:4 (2007) 43-48.

8. M. Williams. "Excitons form superfluid in certain 2-D combos". (2020, June 15) retrieved 19 June 2020 from https://phys.org/news/2020-06-excitons-superfluid-d-combos.html.

9. S.J. Putterman, I. Rudnick. "Quantum Nature of Superfluid Helium". Physics Today 24:8 (1971) 39-47.

10. A. Baggaley, J. Laurie, S. Laizet. "Numerical simulations of thermal counterflow in the presence of solid boundaries". Powerpoint Presentation. University of Glasgow, 2014.

11. S. Balibar. "Laszlo Tisza and the two-fluid model of superfluidity". C. R. Physique 18 (2017) 586-591.

12. University of Innsbruck. "Observation of second sound in a quantum gas". ScienceDaily (15 May 2013) <www.sciencedaily.com/releases/2013/05/130515131508.htm>.

13. R.J. Donnelly. “The Two-Fluid Theory and Second Sound in Liquid Helium”. Physics Today 62:10 (2009) 34-39.

14. J.R. Pellam. "Second Sound Propagation in Liquid Helium". Physics Today 6:10 (1953) 4-9. 